\title{
Tabaco บ salud oral en estudiantes de la Universidad de Santiago de Compostela
}

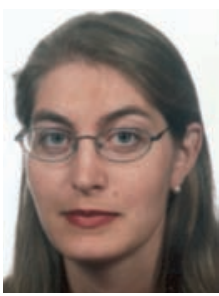

Fernández-Riveiro, Paula

Oral health and tobacco in students at Santiago de Compostela University

\section{Fernández-Riveiro, Paula* Gonzálvez Becerca, Patricia * Leis Fillou, Catherine** smulth Chamosa, Ernesto**}

\section{*Profesora Asociada de Odontología Preventiva y Comunitaria de la USC. **Estudiante de Odontología de la USC \\ ***Profesor Titular de Odontología Preventiva y Comunitaria de la USC. Departamento de Salud Pública. Uni- versidad de Santiago de Compostela.}

\section{Correspondencia}

Paula Fernández Riveiro.

Área de Medicina Preventiva. Facultad de Medicina y Odontología.

Rúa San Francisco s/n. 15705. Santiago de Compostela.

Email:mrpaula@usc.es
Resumen: Objetivo. Describir los hábitos de salud oral y consumo de tabaco en los estudiantes de la Universidad de Santiago de Compostela. Material y métodos. Estudio transversal en estudiantes universitarios mediante encuesta sobre hábitos de salud oral y tabaco, exploración de la cavidad oral y determinación de monóxido de carbono (CO) en aire espirado. Resultados. Se realizaron 1279 encuestas, el $27^{\prime} 5 \%$ a hombres y el $72^{\prime} 5 \%$ a mujeres. La media de edad fue de $21^{\prime} 4$ años. Nueve de cada diez sujetos dice que se lava los dientes después de cada comida y el 8 de cada diez que le dedica más de un minuto a su higiene oral en cada cepillado. El número medio de cepillos dentales utilizados en un año fue superior a 3. Prácticamente el $100 \%$ de los encuestados ha acudido alguna vez al dentista por diferentes motivos. Se realizaron 342 exploraciones bucodentales, en las que se halló un índice CAOD superior a 3 y un índice de restauración próximo al 70\%. Con respecto al hábito de fumar, el 32' 4\% dice que fuma en la actualidad, con una media de 11'15 cigarrillos al día. La edad media de inicio de consumo fue a los 16 años. No se observaron diferencias estadísticamente significativas en ninguna de las variables estudiadas entre fumadores y no fumadores excepto en las tinciones dentarias y en la cantidad de pasta utilizada. La media de CO en aire espirado fue de 12,6 en los fumadores. Conclusiones. En los universitarios hay un elevado grado de concienciación sobre hábitos de salud oral y consumo de tabaco, mayor que en la población general de esa edad. No se observan diferencias en los hábitos ni en el estado de salud oral entre fumadores y no fumadores.

Palabras clave: Salud oral. Tabaco. Estudiantes universitario

Abstract: Objective. The aim of this study was to describe the habits related to oral health and to determine the patterns of tobacco smoking among students at the University of Santiago de Compostela. Material and Methods. Advanced students in dentistry carried out a survey on habits and an examination of the oral cavity of their peers. They also determined CO concentration in exhaled air. Results. The questionnaires were answered by 1279 students, $27.5 \%$ of which were male and $72.5 \%$ female. The mean age of the sample was 21.4 years. Among respondents, $90 \%$ washed their teeth after eating, above $80 \%$ spent more than 1 minute in their oral hygiene and used an average of more than 3 toothbrushes per year. Nearly $100 \%$ of the sample visited the dentist. We performed 342 examinations which resulted in a DMF rate of 3.71 and a restoration rate of $70.8 \% ; 32.4 \%$ of the population smoked an average of 11.5 cigarettes per day. The most frequent starting age of the habit was 16 years-old. No significant differences were found between smokers and non smokers for most of the studied variables with the exceptions of the presence of discolored teeth, the amount of toothpaste used for brushing teeth and the subjective perception of oral health. The average value of expired CO in smokers was 12.6. Conclusions. Among students of our university there is a high degree of awareness concerning oral health. Tobacco smoking habit is less frequent than in the general population in the same age range. There were no differences between smokers and non smokers for oral health and oral health habits.

Key words: Oral health. Tobacco. Undergraduate students.

\section{BIBLID [1138-123X (2007)12:1-2; enero-junio 1-104]}

Fernández-Riveiro P, Gonzálvez Becerra P, Leis Filloy C, Smyth Chamosa E. Tabaco y salud oral en estudiantes de la Universidad de Santiago de Compostela. RCOE 2007;12(1-2):23-29. 


\section{Introducción}

El odontólogo tiene un importante papel como educador sanitario en el ámbito oral y de la salud en general. Entre las actividades de promoción de la salud, se deben incluir actuaciones sobre el abandono del consumo de tabaco y la instauración de hábitos relacionados con la salud, como el control de la dieta y la higiene. El tabaco puede influir negativamente en el área bucal en patologías como el cáncer oral o la enfermedad periodontal, afectando negativamente en la evolución de la mayor parte de los tratamientos dentales', con especial repercusión sobre la salud periodontal y los implantes dentales. Por lo tanto, los odontólogos tienen una función básica en el control del hábito de fumar de sus pacientes y de la comunidad. Se debe registrar en la historia clínica la presencia del hábito y ejercer en la consulta una actividad de educación para la salud con campañas que favorezcan la deshabituación tabáquica del paciente. Es importante que durante la fase universitaria, los futuros odontólogos se formen tanto en actividades de promoción de la salud como en los procedimientos diagnósticos, preventivos y rehabilitadores de la salud oral. Por este motivo y coincidiendo con el Día de la Salud Bucodental, que en el año 2004 estaba dedicado a la «Influencia del tabaco en la salud oral», la Asociación de Estudiantes de Odontología de Santiago (AEOS) y el Departamento de Odontología Preventiva de la Universidad de Santiago de Compostela, pusieron en marcha una jornada de promoción de la salud en casi la totalidad de las facultades de la Universi- dad de Santiago de Compostela (USC) (Campus de Santiago), en la que alumnos de la licenciatura de odontología desarrollaron el trabajo de campo y de promoción de la salud.

\section{Método}

Se realizó un estudio transversal entre los estudiantes universitarios del Campus Universitario de la USC en Santiago de Compostela. Los participantes en este trabajo fueron voluntarios que aceptaron participar en este trabajo. Las facultades en las que se realizó el trabajo se dividieron en Ciencias de la Salud (CS) y No Ciencias de la Salud (NCS): Medicina, Ciencias de la Educación y Enfermería (comparten edificio), Farmacia y Óptica (facultades que agrupamos en lo que denominamos Ciencias de la Salud) y Geografía e Historia, Derecho, Relaciones Laborales y Políticas (comparten edificio), Ciencias de la Comunicación, Ingeniería Química, Ciencias Económicas y Empresariales, Psicología, Biología, Químicas, Matemáticas y Filología (que denominamos en conjunto No Ciencias de la Salud). Para acceder al máximo número de estudiantes, los puntos de estudio se ubicaron en los pasillos de las facultades próximos a las zonas de acceso.

Quince equipos formados por seis alumnos de la licenciatura de odontología, realizaron el estudio en cada una de las facultades; tres estudiantes se encargaron de la realización de las encuestas y los otros tres de la exploración.

El estudio constó de tres partes: en la primera se realizó una encuesta sobre hábitos de salud en la que se incluyeron aspectos relacionados con los hábitos de higiene oral, las consultas al odontólogo y el consumo de tabaco. En la segunda parte se realizó la exploración de la cavidad oral, registrando la presencia de caries, obturaciones y ausencias dentarias, la presencia de placa y de tinciones dentales. La exploración oral fue realizada por los estudiantes del segundo ciclo de la licenciatura de odontología, que previamente a la realización del trabajo de campo, recibieron una formación homogénea sobre los criterios diagnósticos de la $\mathrm{OMS}^{2}$. El paciente y el explorador estaban sentados enfrente, y se empleó espejo y sonda dental con iluminación artificial. La desinfección del material se hizo siguiendo las pautas estándar, de igual modo que la adopción de las medidas de protección universal frente a la infección cruzada en odontología (guantes, mascarillas, paños, etc). En la tercera parte se mide la concentración de los niveles de monóxido de carbono (CO) en aire espirado, mediante el carboxímetro Smokerlyzer ${ }^{\circledR}$ (Bedfont) (www.bedfont.com/smokerlyzer). Esta prueba se registró en seis facultades, debido a la disponibilidad de seis aparatos de medición. El registro de la medición se hizo siguiendo las indicaciones del fabricante: se realizó una inspiración profunda, una pausa de 15 segundos con el aire inspirado y una espiración lenta final. Este método permitió determinar la exposición a las diferentes fuentes de monóxido de carbono de las últimas 48 horas, tanto las fuentes activas (tabaco) como pasivas (ambiente de fumadores, contaminación).

Durante los días en los que se realizó el trabajo de campo, los estudiantes eran abordados por los encuesta- 


\begin{tabular}{|c|c|c|}
\hline Cepillado dientes & Fumadores & No fumadores \\
\hline $1 \mathrm{vez} / \mathrm{día}$ & $9^{\prime} 9 \%$ & $7^{\prime} 3 \%$ \\
\hline Despues cada comida & $88 ' 9 \%$ & $92 \%$ \\
\hline No todos los días & $1^{\prime} 2 \%$ & $0,5 \%$ \\
\hline Nunca & $0 \%$ & $0^{\prime} 2 \%$ \\
\hline
\end{tabular}

\begin{tabular}{|c|c|c|}
\hline Cantidad de pasta & Fumadores & No fumadores \\
\hline cepillo lleno & $44^{\prime} 8 \%$ & $41^{\prime} 2 \%$ \\
\hline 1/2 cepillo & $44^{\prime} 1 \%$ & $41 \%$ \\
\hline $1 / 3$ cepillo & $11^{\prime} 1 \%$ & $17^{\prime} 5 \%$ \\
\hline no usa pasta & $0 \%$ & $0^{\prime} 2 \%$ \\
\hline
\end{tabular}

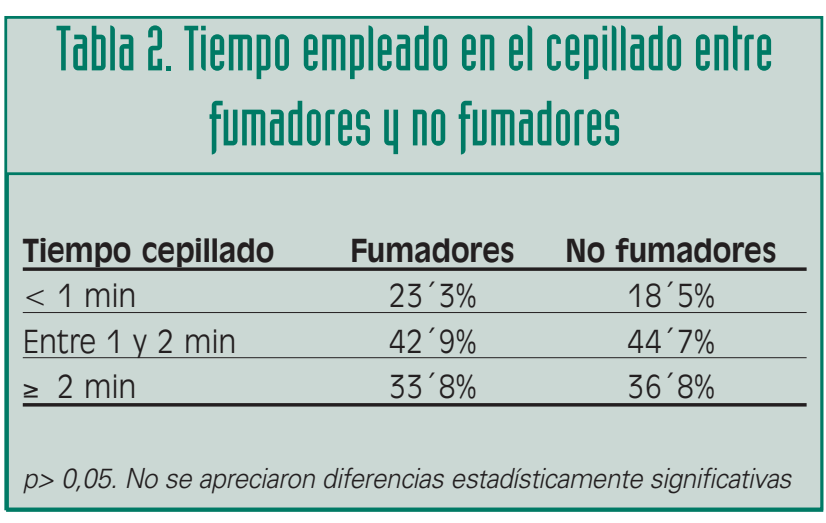

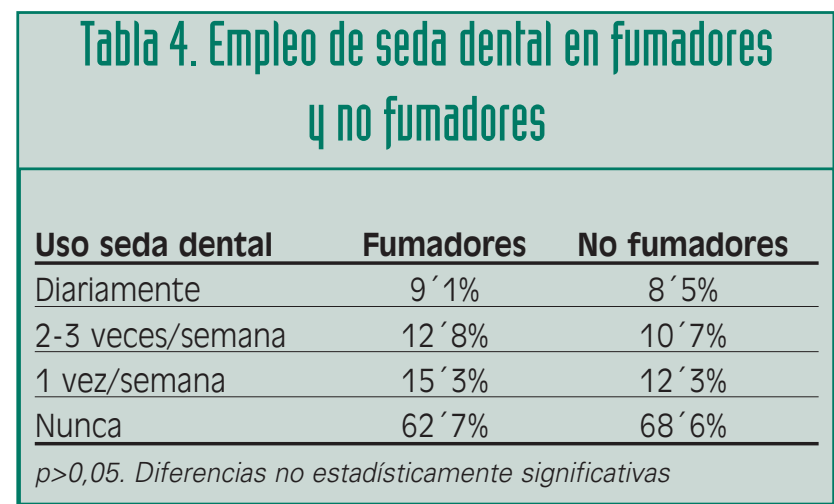

dores, que solicitaban su participación. Si aceptaban, se les entregaba la encuesta y se les realizaba la exploración de la cavidad oral y la determinación del $\mathrm{CO}$ en el aire espirado. Los días de recogida de datos se aprovechó también para entregar información en folletos en los que se aconsejaba el abandono del hábito tabáquico y se informaba sobre su perniciosa influencia sobre la salud oral, a los participantes y a todo el personal de las facultades que se interesara por la campaña.

\section{Resultados}

En total 1341 individuos participaron en el estudio, con un intervalo de edad entre los 17 y los 75 años. Con el fin de tener una muestra más homogénea, se acotó el rango de edad entre los 17 y los 30 años, quedando 1279 individuos, siendo la media de edad de 21'4 años. Se obtuvo una participación del $27^{\prime} 5 \%$ de hombres (347), frente al $72^{\prime} 5 \%$ de mujeres (932).

De los 1279 individuos, un 22'8 \% pertenecían a las facultades de Ciencias de la Salud y un $77^{\prime} 2 \%$ al resto de facultades. La distribución por facultades fue la siguiente: un 8'8\% de Psicología, un 8'6\% de Medicina, un 7\% de Enfermería y Ciencias de la Educación, un 3'8\% de Óptica y un 3'4\% de Farmacia, un 6'5\% de Biología, un $10^{\prime} 9 \%$ de Ciencias de la Comunicación, un 8'4\% de Derecho, un 7’9\% de Ciencias. Económicas y Empresariales, un $7^{\prime} 2 \%$ de Filología, un 6’8\% de Ingeniería Química, un 6’3\% de Matemáticas, un 5'3\% de Relaciones Laborales y Políticas, un 4'8\% de Químicas y un 4 '4\% de Geografía e Historia.

\section{Resultados de la encuesta}

\section{Hábitos de salud oral}

Los hábitos de los sujetos fumadores y no fumadores respecto a la frecuencia del cepillado dental, el tiempo dedicado a cada cepillado, el empleo de pasta dentífrica en el cepiIlo, el uso de seda dental y el empleo de suplementos de flúor se pueden observar en las tablas 1,2,3,4 y 5 . El cepillado en 9 de cada 10 sujetos fue 


\begin{tabular}{|c|c|c|}
\hline Empleo suplementos & Fumadores & No fumadores \\
\hline si & $85 \%$ & $89,4 \%$ \\
\hline no & $15 \%$ & $10^{\prime} 6 \%$ \\
\hline
\end{tabular}

\begin{tabular}{|c|c|c|}
\hline Motivo & Fumadores & No fumadores \\
\hline urgencia & $14^{\prime} 6 \%$ & $13^{\prime} 7 \%$ \\
\hline revisión & $35^{\prime} 8 \%$ & $43^{\prime} 7 \%$ \\
\hline ortodoncia & 7'9\% & $7^{\prime} 8 \%$ \\
\hline limpieza & $9^{\prime} 9 \%$ & $7^{\prime} 8 \%$ \\
\hline sellador, flúor & $1 \%$ & $1 \%$ \\
\hline otros & $9^{\prime} 4 \%$ & $8^{\prime} 8 \%$ \\
\hline varios & $21^{\prime} 5 \%$ & $17^{\prime} 2 \%$ \\
\hline
\end{tabular}

\begin{tabular}{|c|c|c|}
\hline Última visita & Fumadores & No fumadores \\
\hline$<6$ meses & $46^{\prime} 7 \%$ & $46^{\prime} 5 \%$ \\
\hline Entre 6 meses y 1 año & $25^{\prime} 5 \%$ & $26^{\prime} 6 \%$ \\
\hline$>1$ año & $27^{\prime} 7 \%$ & $26^{\prime} 9 \%$ \\
\hline
\end{tabular}

\begin{tabular}{|c|c|c|}
\hline Última limpieza & Fumadores & No fumadores \\
\hline nunca & $24^{\prime} 5 \%$ & $28^{\prime} 8 \%$ \\
\hline$<6$ meses & $26^{\prime} 6 \%$ & $23^{\prime} 6 \%$ \\
\hline 6 meses - 1 año & $24^{\prime} 7 \%$ & $24^{\prime} 8 \%$ \\
\hline 1-2 años & $13^{\prime} 9 \%$ & $12^{\prime} 8 \%$ \\
\hline$\geq 2$ años & $10^{\prime} 3 \%$ & $10 \%$ \\
\hline
\end{tabular}

diario; sin embargo el empleo diario de seda dental lo realizó menos del $10 \%$ de la muestra. La cantidad de pasta dental presentó diferencias en función del hábito de tabaco, utilizando los fumadores mayor cantidad de pasta dental en el cepillo. El número de cepillos gastados en un año superó las 3 unidades $\left(3^{\prime} 59 \pm 2^{\prime} 4\right.$ en fumadores y $3^{\prime} 68 \pm 2^{\prime} 3$ en no fumadores). Los suplementos de flúor fueron usados por más del $80 \%$ de los sujetos.

Las variables relacionadas con las visitas al odontólogo y la salud oral percibida se pueden observar en las tablas 6,7 y 8 . La mayor parte de la muestra había acudido al odontólogo hacía menos de un año, siendo las revisiones el motivo principal por el que acudieron.

Si analizamos la morbilidad bucodental percibida (tabla 9), los fumadores consideran que tienen peor estado de salud bucodental $(p<0,05)$. Sólo el 50 ' $2 \%$ de los fumadores considera tener buena salud oral.

\section{Consumo de tabaco}

El $32{ }^{\prime} 4 \%$ de los sujetos declara ser fumador, sin que se aprecien diferencias entre sexos.

Por facultades, el porcentaje de fumadores en las facultades de Ciencias de la Salud es de un $26 \%$ y de un $34^{\prime} 4 \%$ en las de No Ciencias de la Salud. La edad media de comienzo del hábito fue a los 16 años. Si consideramos sólo los que inician el consumo de tabaco antes de los 18 años, obtendríamos un porcentaje del 61 '3\%. Los estudiantes de las facultades de Ciencias de la Salud que iniciaron el hábito antes de los 18 años asciende al 70 ' $5 \%$ de los estudiantes, frente al $59^{\prime} 4 \%$ de las otras facultades. Existen diferencias en relación al sexo, ya que el $63^{\prime} 7 \%$ de mujeres se inician en el consumo antes de los 18 años, frente a un $54,7 \%$ de los hombres.

La media de cigarrillos fumados se sitúa en unos $11^{\prime} 15$ cigarrillos diarios sin que se apreciasen diferencias apreciables entre los alumnos de 


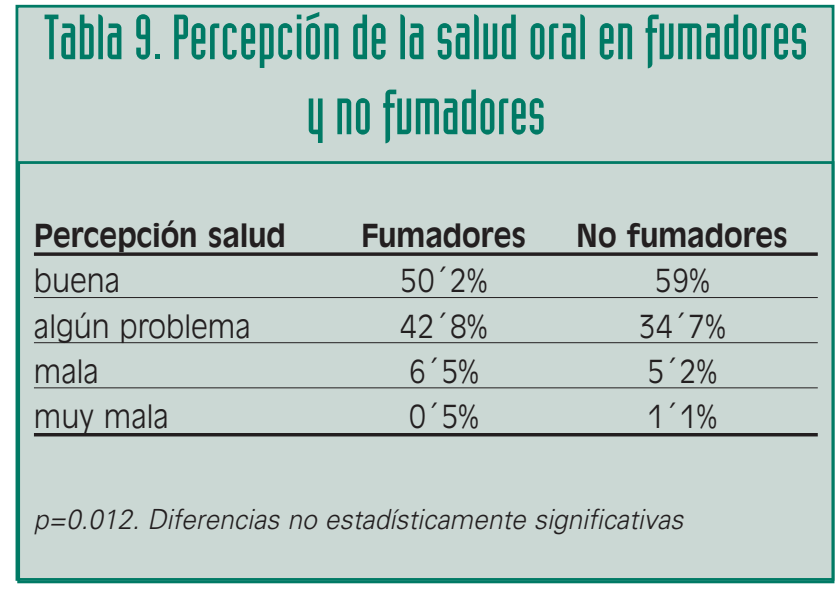

\begin{tabular}{|c|c|c|}
\hline \multicolumn{3}{|c|}{$\begin{array}{l}\text { Tabla ll. Presencia de placa visible según el hábil } \\
\text { labáquico y el tipo de facultad }\end{array}$} \\
\hline $\begin{array}{l}\text { Presencia } \\
\text { de placa }\end{array}$ & $\begin{array}{c}\text { Fumadores/ } \\
\text { no fumadores }\end{array}$ & CS/NCS \\
\hline Placa no visible & $43^{\prime} 9 \% / 48^{\prime} 3 \%$ & $61^{\prime} 2 \% / 41^{\prime} 6 \%$ \\
\hline Algo de placa & $45^{\prime} 8 \% / 41^{\prime} 1 \%$ & $35^{\prime} 3 \% / 46^{\prime} 3 \%$ \\
\hline Mucha placa & $10^{\prime} 3 \% / 10^{\prime} 6 \%$ & $3^{\prime} 5 \% / 12^{\prime} 1 \%$ \\
\hline $\mathrm{p}<0,05^{*}$ & $P>0,05$ & $p=0,004^{*}$ \\
\hline $\begin{array}{l}\text { CS: Ciencias de Sa } \\
{ }^{*} \text { Diferencias estac }\end{array}$ & S: No Ciencias de & la Salud \\
\hline
\end{tabular}

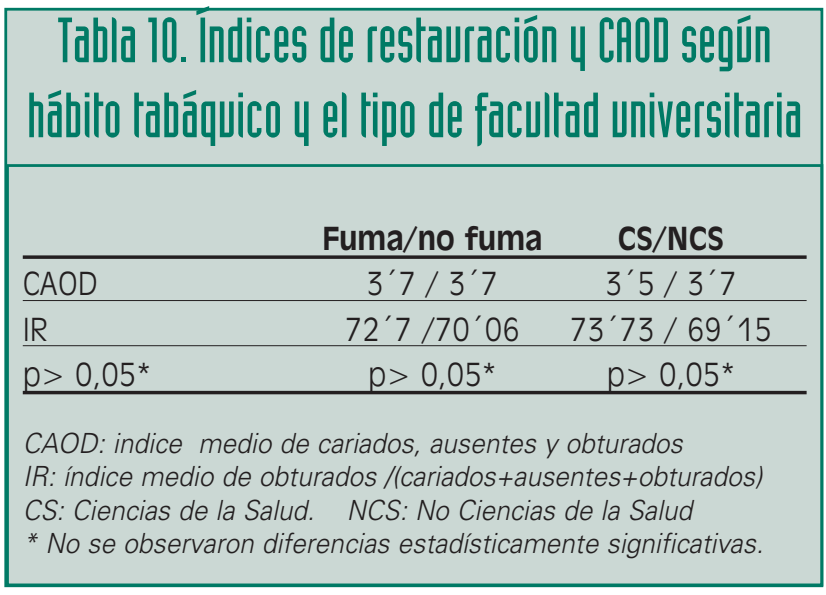

\begin{tabular}{|c|c|c|}
\hline \multicolumn{3}{|c|}{$\begin{array}{l}\text { Tabla la. Tinciones dentales } \\
\text { en fumadores u no fumadores }\end{array}$} \\
\hline Tinciones & Fumadores & No fumadores \\
\hline ninguna & $54^{\prime} 7 \%$ & $66^{\prime} 9 \%$ \\
\hline tetraciclinas & $0 \%$ & $1^{\prime} 8 \%$ \\
\hline fluorosis & $0.9 \%$ & $0,6 \%$ \\
\hline Tabaco & $16 \%$ & $1^{\prime} 2 \%$ \\
\hline otras & $28^{\prime} 3 \%$ & $29^{\prime} 5 \%$ \\
\hline
\end{tabular}

Ciencias de la Salud y el resto de facultades. El $37^{\prime} 6 \%$ de los nombres fuman mas de 20 cigarrillos al día frente al $29 \%$ de la mujeres.

El 23'9\% estaría dispuesto a dejar de fumar ahora mismo, mientras un $63^{\prime} 4 \%$ lo haría en el futuro y un $12^{\prime} 7 \%$ no lo dejaría (el $17 \%$ de los hombres fumadores y el $12 \%$ de la mujeres). El $58^{\prime} 7 \%$ de estos fumadores afirma haber intentado dejarlo en alguna ocasión $151^{\prime} 9 \%$ hombres y $61^{\prime} 1 \%$ mujeres), frente a un $41^{\prime} 3 \%$ que nunca lo ha intentado.

Un $94^{\prime} 2 \%$ de los encuestados consideró que el tabaco influye negativa- mente en su salud oral, un 3'9\% opina que no existe relación y un $1^{\prime} 9 \%$ no sabe.

\section{Determinación de monóxido de carbono (CO)}

El registro con el carboxímetro del Co espirado se realizó en 137 sujetos de 6 facultades (54\% eran de ciencias de la Salud, $65 \%$ eran mujeres y el $61 \%$ eran fumadores). Se obtuvo un valor medio de la concentración de $\mathrm{CO}$ en toda la muestra de $9^{\prime} 09 \pm 8^{\prime} 6$, oscilando los valores entre 0 y 42 . No se observaron diferencias entre sexos pero sí una menor concentración de CO en los alumnos de facultades de Ciencias de la Salud ( $\left.8^{\prime} 25\right)$ que en los de No Ciencias de la Salud (10'11). Entre fumadores y no fumadores las diferencias fueron significativas $\left(p<0^{\prime} 005\right)$; los fumadores tuvieron una concentración de $12^{\prime} 6$ de Co espirado frente al $3^{\prime} 56$ de los no fumadores.

\section{Exploración oral}

Se exploraron 361 individuos, 114 hombres $(32 \%)$ y 247 mujeres (68\%). Se determinó un índice CAOD de 3'7 . El índice de restauración, el CAOD, el grado de higiene oral y las tinciones se pueden ver en las tablas 10 y 11 . Se 
observó una menor cantidad de placa en los alumnos de las facultades de Ciencias de la Salud frente a los de No Ciencias de la Salud. En la exploración de tinciones (tabla 12), los fumadores presentaron, un mayor porcentaje de tinciones debidas al hábito tabáquico ( $16 \%$ frente a un $\left.1^{\prime} 2 \%\right)$

\section{Discución}

El presente estudio nos permite analizar el hábito tabáquico y el estado bucodental de una muestra de estudiantes de la Universidad de Santiago de Compostela. Cabe mencionar las limitaciones en la selección de la muestra del presente estudio, ya que no se obtuvo al azar. Está formada por los alumnos que acudieron el Día de la Salud Bucodental a las facultades donde se hizo el estudio y decidieron voluntariamente participar en él, por lo que los alumnos que no se hallaban en esas facultades en esas momento y los que no quisieron participar podrían sesgar los resultados obtenidos.

No se han hallado estudios en población universitaria en los que se traten a la vez las variables de hábitos de higiene oral, consumo de tabaco y estado de la cavidad oral, dificultando el poder contrastar los resultados.

En lo que respecta a los resultados obtenidos sobre los hábitos de higiene oral, en relación a la frecuencia del cepillado, obtenemos una mayor frecuencia en nuestra muestra que en otros estudios; en los universitarios de Santiago el $90 \%$ afirma cepillarse los dientes unas 3 veces al día mientras que en población general, mayor de 14 años de España ${ }^{3}$ la frecuencia se reduce al 30\%. En un estudio similar en estudiantes de Medicina y Odontología de la Universidad de Barcelona ${ }^{4}$ se observó que entre el 20 y el $40 \%$ de los estudiantes se cepillaban tres veces al día. Sobre el tiempo dedicado al cepillado en esta Universidad catalana, un $40 \%$ le dedica más de dos minutos, dato muy similar al obtenido en nuestro estudio, que es de un $36^{\prime} 1 \%$.

Con respecto al número de cepillos gastados al año por individuo, obtuvimos una media de 3 cepillos por universitario de Santiago de Compostela, mientras que en la población general no llega a 1 cepillo por año ${ }^{3}$.

Sobre el uso o no de seda dental, los datos obtenidos son similares a los encontrados en otros estudios, como en el estudio prospectivo Delphi en España en el que el 78,5\% de los sujetos encuestados afirman no usar nunca seda dental y el $93^{\prime} 5 \%$ no utilizan nunca los cepillos interdentales ${ }^{5}$. En general en España ${ }^{3}$ el $78 \%$ de la población no emplea seda dental, porcentaje muy similar al hallado en nuestro estudio (un $70 \%$ de los universitarios del estudio no la utiliza).

En lo que respecta a los datos obtenidos sobre consumo de tabaco, los resultados se aproximan a los de otros estudios con estudiantes universitarios. Así, en el estudio de consumo de tabaco en estudiantes de sexto curso de medicina de España ${ }^{6}$ se obtuvo un porcentaje de fumadores próximo al $30 \%$, una media de 11 cigarros al día y una edad media de inicio de consumo de 17 años. En el estudio encuesta sobre tabaquismo en estudiantes universitarios que practican ejercicio físico $(8,9)$ observaron una prevalencia de fumadores del 30\% y una media de 11 cigarrillos diarios. Este porcentaje es inferior al hallado en 1987 en un estudio con estudiantes de Medicina de la Universidad de Santiago de Compostela en el que se halló un $61^{\prime} 9 \%$ de fumadores que consumían un promedio de 16 cigarrillos al día?.

Cabe mencionar las diferencias entre nuestra muestra, que incluye estudiantes de diferentes carreras y la de otros estudios, que se centraban en carreras de ámbito sanitario. En estos estudios, al igual que en el nuestro, también se observó un porcentaje elevado de estudiantes (22$30 \%)$ que se iniciaron en el hábito tabáquico en la universidad, lo que debe ser tenido en cuenta para llevar a cabo estudios y programas preventivos del hábito tabáquico en ambiente universitario.

Del mismo modo es importante la frecuencia del hábito en las distintas facultades. En otros estudios consultados $^{10}$ se observó la misma tendencia que hallamos en nuestra muestra, en la que existe una mayor proporción de fumadores en las facultades de No Ciencias de la Salud, siendo Psicología, Historia y Geografía las facultades donde más se fuma.

Con respecto a los resultados de la exploración dental, dado que el rango de edad de nuestra muestra es de 17 a 30 años, tomaremos como referencia los valores de población de 15 y 35- 44 años de la Encuesta de Salud Oral en España del 200511. En la encuesta del 2005 la población de 15 años presentaba un índice CAOD de 2'18, que es inferior al obtenido en el presente estudio con universitarios (CAOD 4) e inferior al de la encuesta del 2005 para 35-44 años (9'61) En el índice de restauración observamos 
alguna diferencia entre las dos muestras; en la población de 15 años del estudio estatal del 2005 este índice fue del $59^{\prime} 6 \%$, y del $54 \%$ en el tramo de edad 35-44, mientras que en este estudio se alcanzó un 70\% de los dientes afectados restaurados.

En este trabajo, por cuestiones técnicas, no se ha podido evaluar el estado de salud periodontal de los universitarios pero en trabajos próximos y una vez que ha entrado en vigor la Ley 28/2005 frente al tabaquismo, sería un aspecto interesante a analizar.

En cuanto a la percepción subjetiva de los individuos de la muestra sobre el estado de salud bucodental $(58 \%$ la considera buena) es peor que la percepción que recoge la ENS del 2003 en la que el $79 \%$ de los sujetos consideran que su boca está sana ${ }^{12}$.
En esta encuesta12 también se observó un incremento del 32'5\% (1987) al 61\% (2003) de consultas al dentista motivados por una revisión general. En nuestro trabajo el 41'6\% acudió la última vez al dentista por revisión, junto a un $8 \%$ que acudió por una limpieza y otro 8\% por ortodoncia. Estos datos reflejan una tendencia a acudir a consulta por motivos de prevención y mantenimiento de la salud, disminuyendo el porcentaje de sujetos que acuden por dolor bucodental.

\section{Conclusiones}

Se ha observado una elevada motivación hacia los hábitos de higiene oral, con frecuencias de cepillado den- tal diario próximos al 90\% y ausencia de placa dental en la exploración en prácticamente la mitad de los individuos. Es llamativo el escaso empleo de seda dental. Sólo la emplea habitualmente uno de cada cinco universitarios del estudio.

También se observó una elevada motivacion de asistir a la consulta del odontólogo periódicamente; uno de cada dos universitarios entrevistados había acudido hacía menos de seis meses.

En relación al hábito tabáquico, el consumo es similar al de la población general. El consumo de tabaco no influyó en la mayoría de las variables empleadas en este estudio excepto en las tinciones, en la cantidad de pasta dental empleada en el cepillado y en la percepción de la salud oral.

\section{Bibliografia recomendada}

Para profundizar en la lectura de este tema, el/los autor/es considera/an interesantes los artículos que aparecen señalados del siguiente modo: *de interés ${ }^{*}$ de especial interés.

1. Petersen PE.Tobacco and oral health: the role of the World Health Organization. Oral Health Prev Dent. 2003;1:309-315.

2. WHO: Oral Health Surveys. Basic Methods, ed $4^{\circ}$. Geneva, 1997.

3. Control de placa e higiene bucodental. $1^{\circ}$ Workshop Ibérico. Editor Mariano Sanz. Ed Ergon. Madrid, 2003

4. Nevot González MC, Cortés Martinicorena FJ, Cuenca Sala E. Hábitos y actitudes de salud oral en estudiantes de odontología y medicina de la Universidad de Barcelona. Arch Odontoest. 2001;17:465-477.

5. Estudio prospectivo Delphi. Libro blanco. La salud bucodental en España. Odonto-estomatología 2005. Barcelona, 1997.

6. Mas A, Nerón I, Barrueco M, Cordero J, Guillén D, Jiménez-Ruiz C y Sobradillo V. Consumo de tabaco en estudiantes de sexto curso de medicina de España. Arch Bronconeumol. 2004;40:403-408.

7. Gestal-Otero JJ, Montes-Martínez A. Smoking habits of final-year galician medical students. Rev Epidem Santé Publ. 1987, 35:386-392.

8. Nerín I, Crucelaegui A, Novella P, Ramón y Cajal P, Sobradiel N, Gericó R. Encuesta sobre tabaquismo en estudiantes universitarios en relación con la práctica de ejercicio físico. Arch Bronconeumol. 2004; 40:5-9.

9. Valdés-Salgado R, Micher JM, Hernández L, Hernández M, Hernández-Ávila M. Tendencias del consumo de tabaco entre alumnos de nuevo ingreso a la Universidad Nacional Autónoma de México, 1989 a 1998 . Salud pública México 2002;44:S44-S53.

10. Villar Hoz E, Viñas Poch F, Juan Ferrer J, Caparrós Caparrós B, Pérez Guerra I y Cornellà Canals M. Dimensiones psicopatológicas asociadas al consumo de tabaco en población universitaria. Ana Psicología. 2004;20:33-46

11. Bravo-Pérez M, Casals-Peidró E, Cortés-Martinicorena FJ, Llodra-Calvo JC. Encuesta de salud oral en España 2005. RCOE 2006; 11:409-456

12. Ministerio de Sanidad y Consumo. Encuesta Nacional de Salud. Madrid, 2003.

\section{Artículos destacados}

- Petersen PE. Tobacco and oral health: the role of the World Health Organization. Oral Health and Preventive Dentistry. 2003;1(4):309-315. El programa de salud oral de la OMS da priori- dad al control del tabaco e incorpora la salud oral en el programa. Resalta la negativa influencia del hábito tabáquico sobre patologías orales como el cáncer oral, la enfermedad periodontal, la halitosis, la aparición de defectos congénitos como el labio leporino y la hendidura palatina en hijos de madres fumadoras durante el embarazo, alteraciones en la cicatrización de los tejidos orales y depresión del sistema inmune de defensa contra las infecciones. El programa de la OMS incorpora la labor de asociaciones dentales y profesionales de la salud oral en la prevención del consumo de tabaco por su importante papel comunicador con los pacientes y las comunidades.

- Gestal-Otero JJ, Montes-Martínez A. Smoking habits of final-year galician medical students. Rev Epidem Santé Publ. 1987, 35:386-392.

Estudio del hábito de consumo de tabaco a través de un cuestionario en estudiantes de Medicina de la Universidad de Santiago de Compostela en la década de los 80 . La prevalencia de exfumadores fue baja y la de fumadores alta $\left(67^{\prime} 9 \%\right)$ con una media de 16 cigarrillos al día consumidos. El $60 \%$ de los fumadores habían adquirido el hábito entre los 14 y los 17 años. 